\title{
THE INFLUENCE OF POWDER SIZE AND PACKING DENSITY ON THE TEMPERATURE DISTRIBUTION IN SELECTIVE LASER MELTING
}

\author{
Diego Augusto de Moraes \\ Lassonde School of Engineering \\ York University \\ Toronto, Canada \\ moraes@yorku.ca
}

\author{
Aleksander Czekanski \\ Lassonde School of Engineering \\ York University \\ Toronto, Canada \\ alex.czekanski@lassonde.yorku.ca
}

\begin{abstract}
Metal powder properties in Selective Laser Melting (SLM) is among one of the most important factors when implementing new alloy developments for the equipment. In fact, not all commercially available metal powder alloys are ready to be implemented without a comprehensive set of tests. Besides the powder properties, we have a large number of building and environmental parameters that demands extensively research prior implementation. Although selected alloys are commercially available and documented to be used in SLM, including Ti6Al4V, SS316L and In718, the majority of it still not ready to be utilized in this system. The focus of this study is to use a thermal model in order to predict the thermal distribution of the process regarding different aspects of the powder properties, especially the thermal conductivity, when different powder packing densities and diameters are used. A Stainless Steel 304L will be utilized in this work, since it is not yet available to be commercially used. The main goal is to show the capabilities of the Finite Element Method in the pre-definition of optimal parameters for the process using a new alloy development. Our findings can be used as a pre-evaluation guideline when printing SS304L, since the comparison with similar experimental work in the field showed significant resemblance and outcomes. The temperature distributions show that the packing density has greater sensibility on the final temperature distributions, compared to the powder diameter variance. Two different power inputs are compiled and the temperature outcomes demonstrate that a power input of 100 Watts is recommended to use when printing SS304L, rather than 400 Watts that brings high temperature into the powder bed.
\end{abstract}

Keywords-component; Additive Manufacturing Modeling, Selective Laser Melting, Powder Compaction, Effective Thermal Conductivity, Finite Element Method

\section{INTRODUCTION}

Selective Laser Melting (SLM) is an Additive Manufacturing process that utilize metal powder in order to build full dense parts in a layer fashion. Currently, the technology is commercially distributed by few manufacturers

NSERC/Quanser Chair in Design Engineering program around the world, with a selection of alloys available, including Titanium, Stainless Steel, Aluminum, Cobalt-Chrome and Nickel-based alloys [1][2].

The fact that SLM uses a lower space, entitled bed, which is filled with fine metal powder, resulted in the association of this process with the Powder Bed Fusion (PBF) classification. Among them are Direct Laser Melting Sintering (DLMS), Electron Beam Melting (EBM) and Selective Laser Sintering (SLS). It is important to emphasize that all these processes were developed by different entities and timelines, and although some similarities, they have quite different set of parameters and mode of operations [3].

The main objective of this study is to demonstrate the high capability of FEM in this field, utilizing for that a low computational cost thermal model able to predict the temperature distributions when scanning a single layer and track of the powder bed. Some of the aspects of discrete method will be implemented, however only in the thermal conductivity implementation method. We start with the laser power input modeling, by using double-ellipsoidal as the heat flux input into the FEM model. Next the material is modeled, considering the state (solid, liquid, powder) and the temperature effects. Special attention is devoted to the thermal conductivity of the powder and for that a special effective thermal conductivity formulation is used to determine the variance in all conditions (packing density and powder diameter). Important devotion is given to the phase change and emissivity of powder as well. Finally, the model is built and compiled, leading to the results and analysis sessions. The powder diameter and the packing density are the main focuses here, in order to determine the impact on the temperature profile during two set of power input sources.

\section{EFFECTIVE Thermal CONDUCTIVITY OF POWDER BED}

The contact of two adjacent spherical particles is essential when calculating the thermal conductivity, since the major heat exchange is done through conduction [4]. The respectively radius of contact is calculated by the Hertz theory applied for contact interaction of solid bodies [5]. This contact area is very small, especially in the present study, since no plasticity is 
considered and the only force acting among particles are gravity forces.

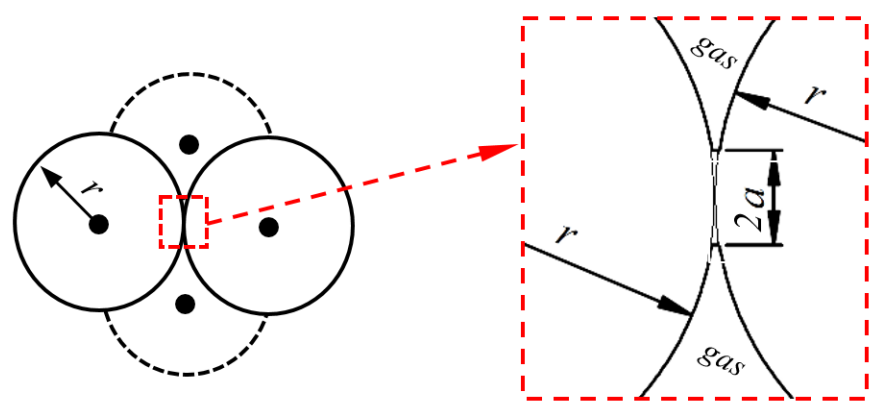

Figure 1 - Particles in contact (right) and the contact region (left)

The material used in the model is Stainless Steel 304L. This alloy has considerably low cost and yet still not available for the process in the industry. The data is extracted from Choong report, including Specific heat, density and the thermal conductivity, which are specified as temperature-dependent properties, essential when simulating high change in temperature distribution over small time increments [6]. To accommodate the phase transformation, the latent heat is included as well, which will modify the capacity heat during the powder melting [3].

The packing density affects the contact radius, consequentially influencing the emissivity and finally the final effective thermal conductivity of the powder bed. Below are the porosity-dependent emissivity for all packing densities and the final effective thermal conductivity of all cases from $300 \mathrm{~K}$ until $T_{\text {Solidus }}$ of SS304L [7].

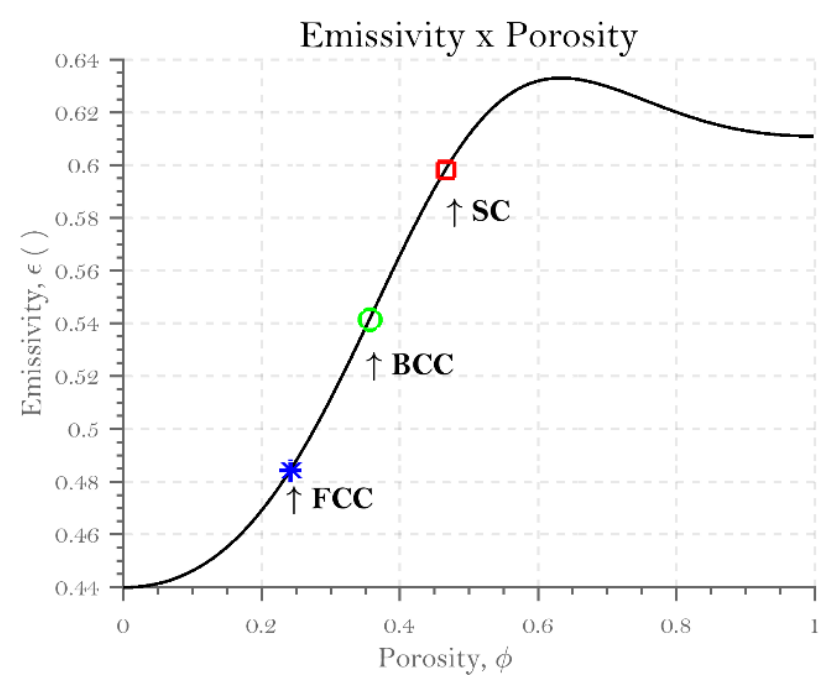

Figure 2 - Porosity-dependent emissivity of SC, BCC and FCC for SS304L [7]

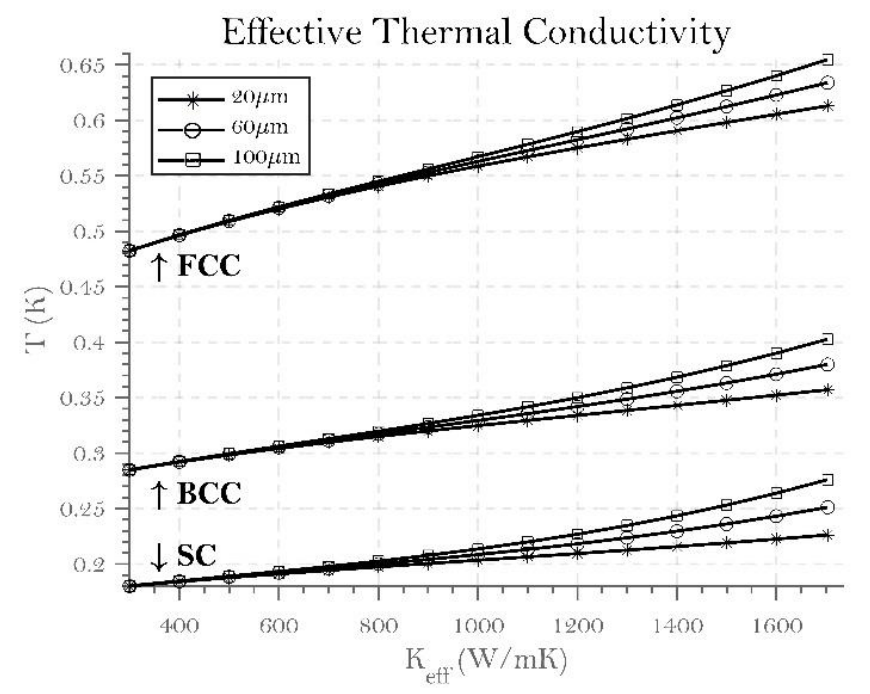

Figure 3 - Effective thermal conductivity of powder for SC, BCC and FCC with 20,60 and $100 \mu \mathrm{m}$ of powder diameter for SS304L [7]

In this study, it is considered three different packing densities, SC, BCC and FCC, with porosity of $0.2424,0.3571$ and 0.467 respectively, along with three powder diameters analysed, 20, 60 and $100 \mu \mathrm{m}$. On previously work the effects of powder packing in the bed was not analyzed and a general value of 0.400 in porosity was adopted [3]. The thermal radiation term is highly influenced by the powder diameter, temperature and emissivity of the powder bed. In the other hand, the thermal conductivity due the contact between particles will vary according to the contact ratio and the initial solid thermal conductivity, since the value of the fractional contact area, $\Lambda$, is less than $3 \times 10^{-4}[7]$.

It is noticeable that the powder diameter does not affect significantly the value of $k_{\text {eff }}$ for temperatures between $300 \mathrm{~K}$ and $800 \mathrm{~K}$. However, for higher temperatures, due the radiation term, there is a perceptible difference. For FCC, as example, there is around $7 \% k_{\text {eff }}$ increase when using $100 \mu \mathrm{m}$ instead $20 \mu \mathrm{m}$. The margin increases to $22 \%$ when doing the same comparison with SC, due the higher emissivity of SC compared to FCC. When comparing between the different packing densities, the $k_{\text {eff }}$ increase margin is even bigger, reaching up to $267 \%$.

\section{Thermal Modeling OF SLM Heat Source}

The thermal model is defined in a way to handle different material phase (liquid, solid and powder state), as well for their variance during different temperatures. A three-dimensional model is established using DC3D8 hexahedron element with $25 \mu \mathrm{m}$ of size and a total domain of $1500 \times 1500 \times 75 \mu \mathrm{m}(1.5 \times 1.5 \mathrm{x}$ $0.075 \mathrm{~mm}$ ). A small domain is justifiable, given the small laser spot diameter, $50 \mu \mathrm{m}$, used in the model and the very low thermal conductivity present on the bed.

The material is modeled as temperature and state dependent, allowing to proper account more accurately the temperature 
distributions. There are some considerations that were made to preserve a low computational time/cost.

\section{Principal assumptions:}

- thermo-fluid effects were not considered, so Marangoni effect and fluid flow in the melt pool is not accounted;

- vaporization of the material is not implemented as well, but the phenomena only occur when utilizing considerably high laser power input $(P=400 \mathrm{~W})$;

- a single track of the laser is observed and the influence of the hatch distance is neglected in order to only focus on the effect of the packing structure and particle diameter in the temperature distributions.

- only average powder (particles) diameter is considered when calculating the effective thermal conductivity of powder state.

ABAQUS is the software used to perform the analysis, since its subroutines allow to model the moving heat source and keep track of the material state. The subroutines DFLUX and FILM were implemented in this study for Gaussian heat flux and convection/radiation definition respectively. The solution given is the temperature distribution of the process, described as $T(x, y, z, t)$. The problem is described by the three dimensional heat conduction equation (8), with the respective manufacturing and chamber environmental boundary conditions $[3,7]$.

\section{RESUlts AND Discussion}

The numerical analysis was conducted for two laser power input (100 and 400 Watts), combined with three packing densities and three different powder diameters, giving 18 compiled result models that ran for 9,5 hours in total (average of approximately $30 \mathrm{~min}$ for each simulation). The temperature distribution was acquired in four central nodes through the thickness located in the middle of the powder bed.

The contact powder and substrate is neglected, however the pre-heat temperature from the substrate is entered as a boundary condition, with a defined pre-defined temperature of $643 \mathrm{~K}$. We have found that different powder diameters within the same packing density have no impact in the final temperature distributions on the model. However, between the three different packing densities the temperature differs. When using $\mathrm{P}=100 \mathrm{~W}$ (Figure 10), the maximum temperature achieved is around $1575 \mathrm{~K}$ at the surface, not enough to reach the melting point of the alloy utilized. However, it is essential to consider that powder can still melt in certain amount due necking between the small particles. The only concern here is at $-75 \mu \mathrm{m}$, which achieved a temperature of $1100 \mathrm{~K}$.

On the same temperature distribution plot, is noticeable that the temperature raises at the same rate for all the packing densities, but as soon as the temperature reaches the maximum amount and the laser scan moves forward, the temperature loss differs from each of the configurations. On the highlighted secondary plot in the same graph, it is shown that SC loses less heat than BCC and FCC. This is justifiable by the fact that SC has a smaller effective thermal conductivity among all, thus exchanging less heat. The biggest difference reaches up to $45 \mathrm{~K}$ between SC and FCC at the surface of the powder bed, representing $3 \%$.

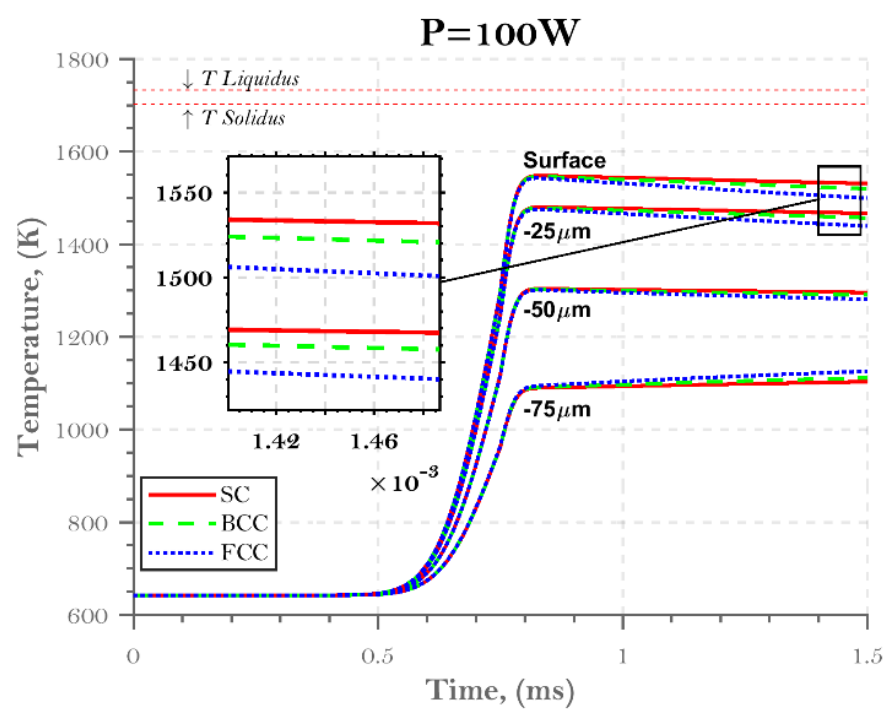

Figure 4 - Temperature distribution of Power input of 100W [7]

Another feature from the first analysis is the fact that the rate of change is very small after the temperature reaches its maximum. This happens because of the domain still on powder state, thus having low thermal conductivity overall. It is important to notice as well that the effective thermal conductivity, although not validated experimentally, followed the same trend experienced by the study of Alkahari et al [23]. In their study the thermal conductivity of SS316L was measured by laser flash technique and the outcomes show a tendency of superior thermal conductivity of $100 \mu \mathrm{m}$ particles when comparing to $20 \mu \mathrm{m}$, the same tendency happens when increasing the packing density.

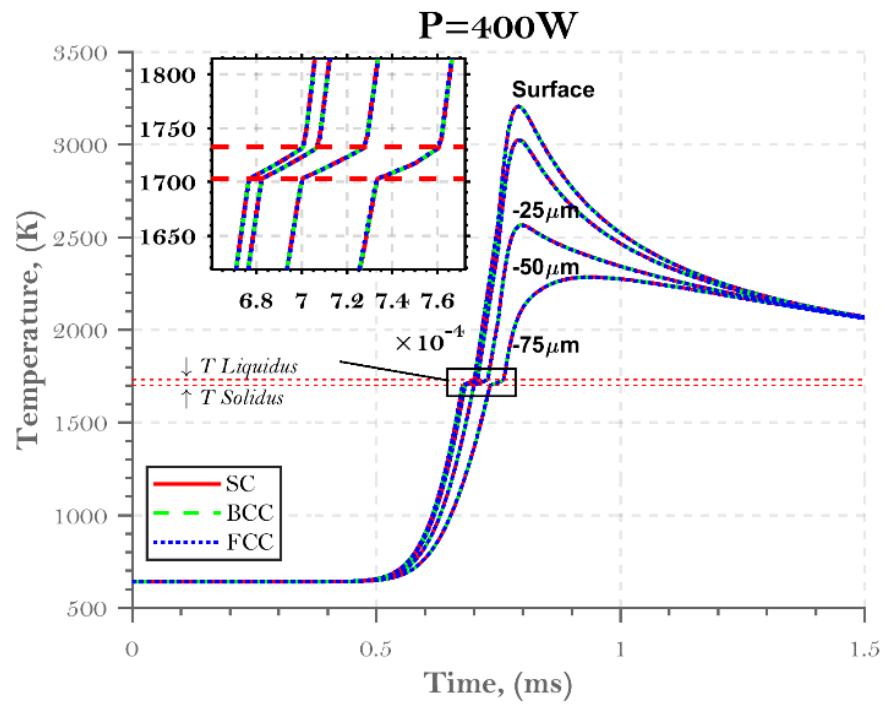

Figure 5 -Temperature distribution of Power input of $400 \mathrm{~W}$ [7]

The second power input $(\mathrm{P}=400 \mathrm{~W})$ is the one which the whole depth of the powder bed layer thickness reaches the 
melting point. However, the maximum temperature achieved at the surface is around $3250 \mathrm{~K}$, which is an undesirable condition that can lead to evaporation of the metal [8]. The bottom reaches around $2100 \mathrm{~K}$, which will most likely induce the melting of some area of the substrate or increase the heat affected area of the same. It is noteworthy here to observe that a gradient of around $1150 \mathrm{~K}$ between the top and bottom layer is induced, whereas at $\mathrm{P}=100 \mathrm{~W}$ the gradient is $475 \mathrm{~K}$. This fact alone is extremely important when analysing residual stresses during SLM. The higher temperature gradient and respectively the cooling rates induced by the process, higher is the residual stresses occurrence.

In the secondary plot of Figure 12, it is highlighted the melting point area between solidus and liquidus temperature (dashed red line). The change of rate of temperature during this interval proves the model is capable of interpreting the different properties states and the phase change by the latent heat of fusion, even with a small time incrementation used.

Furthermore, the results show a very close behaviour with the experimental studies using SS304L found in the literature. Abd-Elghany and Bourel had performed the closest testing to our set-up. They have conducted tensile specimens printing using a machine with $100 \mathrm{~W}$ and $30 / 50 / 70 \mu \mathrm{m}$ layer thickness, concluding that higher layer thickness produced low density and poor mechanical properties specimens [9]. This can be used as an reference to our set-up of $\mathrm{P}=100 \mathrm{~W}$ and layer thickness of $75 \mu \mathrm{m}$, which the laser input was not enough to bring the temperature to the melting point, thus consolidation between powder particles are poor.

\section{CONCLUSIONS}

In this research it is proposed a low cost FEM thermal model capable of measure temperature distributions through the powder bed and consequentially the temperature gradient, of a single layer and track of the laser scanning using SLM process. Although the packing density and powder diameter impact the final value of effective thermal conductivity of the powder bed, the only considerable effects in the temperature distribution was observed for lower power input $(\mathrm{P}=100 \mathrm{~W})$ and only for different packing densities. The reason why the powder diameter did not show major impact in the model is related to the minor effective thermal conductivity change regardless of the diameter and the fact that the model is based in the total volume occupied by the particles as a whole.

The significance of the results demonstrate that a proper compaction and density distribution of the powder bed is important to keep the cooling rates and temperature distributions within the desirable range, however these impacts are only perceivable outside the melt pool zone. In the other hand, by looking to the power input, we can see that $400 \mathrm{~W}$ with the current speed of $1 \mathrm{~m} / \mathrm{s}$ induce high temperature at the surface and through the layer thickness, very close to evaporation point.
This by itself brought a temperature gradient of around $1150 \mathrm{~K}$ through the layer thickness, which is the main cause of warping and residual stresses. For $\mathrm{P}=100 \mathrm{~W}$ the temperature did not reach the melting point at all, which could affect in the consolidation of the particles and in the final porosity of the built part.

Future research is necessary in order validate the model experimentally using different powder diameters and measure on which extent the powder bed is compacted or not.

\section{ACKNOWLEDGMENT}

This research is supported by Lassonde School of Engineering and NSERC/Quanser Chair in Design Engineering program.

\section{REFERENCES}

[1] "SLM Solutions Group AG" [Online]. Available: https://slm-solutions.com/. [Accessed: 30-Dec-2017].

[2] "Renishaw Manufacturing Systems" [Online]. Available: https://renishaw.com/. [Accessed: 30-Dec2017].

[3] de Moraes, D. A., 2017, "Thermal Modeling of 304L Stainless Steel for Selective Laser Melting: Laser Power Input Evaluation," International Mechanical Engineering Congress and Exposition, ASME, Tampa, pp. 1-10.

[4] Sumin Sih, S., and Barlow, J. W., 2004, "The Prediction of the Emissivity and Thermal Conductivity of Powder Beds," Part. Sci. Technol., 22(3), pp. 291304.

[5] Johnson, K. L., 1985, Contact Mechanics, Cambridge University Press, Cambridge.

[6] Kim, C. S., 1975, Thermophysical Properties of Stainless Steels, Argonne National Laboratory.

[7] de Moraes, D., and Czekanski, A., 2018, "Parametric Thermal FE Analysis on the Laser Power Input and Powder Effective Thermal Conductivity during Selective Laser Melting of SS304L," J. Manuf. Mater. Process., 2(3), p. 47.

[8] Fuerschbach, P. W., Norris, J. T., He, X., and Debroy, T., 2003, Understanding Metal Vaporization from Laser Welding, Albuquerque.

[9] Abd- Elghany, K., and Bourell, D. L., 2012, "Property Evaluation of 304L Stainless Steel Fabricated by Selective Laser Melting," Rapid Prototyp. J., 18(5), pp. 420-428. 